\title{
Development of digital design and modelling approaches with preliminary estimation methods based on a digital twin
}

\author{
Andrey Novikov ${ }^{1}$, Elena Zhilenkova ${ }^{2}$, Zorigto Baldanov ${ }^{2}$, and Anton Zhilenkov,** \\ ${ }^{1}$ Saint Petersburg State Marine Technical University, 190121, St. Petersburg, Russia \\ ${ }^{2}$ Peter the Great St. Petersburg Polytechnic University, 195251, St. Petersburg, Russia
}

\begin{abstract}
Approaches to digital design and modeling are investigated. The methods for preliminary estimation of the parameters and structure of a product being created based on a digital twin are proposed. The principle of choosing the characteristics of replaceable modules for a designed object at the stage of research design is shown. The requirements of the nature, placement and application of the target payload were taken into account. It is shown that in research design based on a digital twin, the critical challenge is to jointly optimize the characteristics of all modules and the overall configuration of the object.
\end{abstract}

\section{Introduction}

An object under design is considered as an integral system, consisting of two subsystems:

- carrying platform;

- a complex of payload modules (hereinafter referred to as modules).

A module is understood as a structural element included in the object's information system, designed to place the target payload [1-3].

The characteristics of these two subsystems are closely interdependent. When optimizing the characteristics of complex technical objects, it is necessary to provide for the possibility of changing the functional tasks of the modules based on the variation of the assigned tasks, as well as modernization reserves. The reason for this is that the life cycle of the "basic platform" is much longer than that of the module. This is due to the use of replaceable payload modules, rather than stationary systems and installations [2, 3].

The considered design stage is a research design. At this stage the concept of the object is justified and the project of its exact characteristics is being developed. Models the details of which correspond to the information of the research design stage are used (Fig.1).

\footnotetext{
* Corresponding author: zhilenkovanton@gmail.com
} 


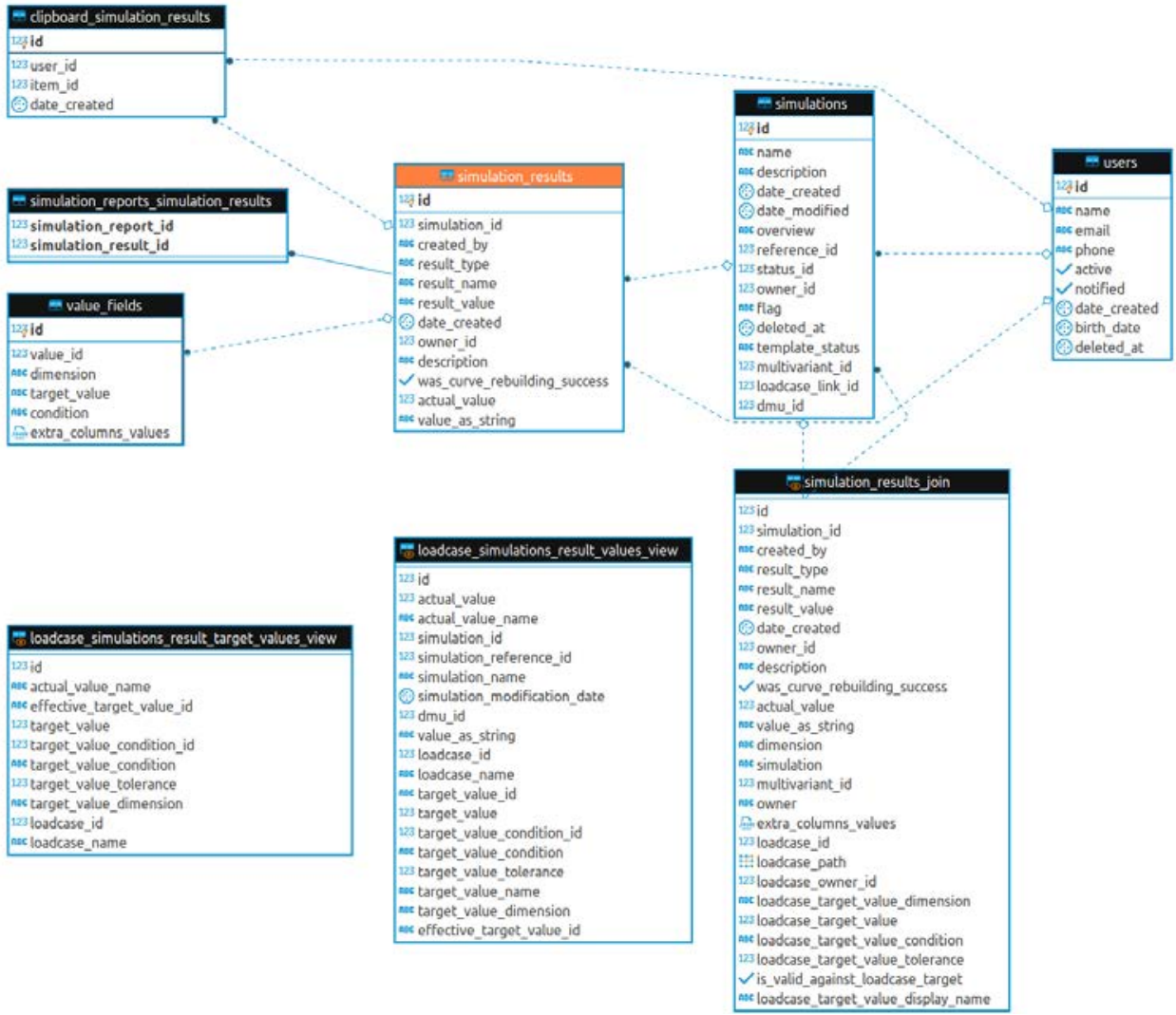

Fig. 1. Possible structure of a digital platform subroutine.

\section{Structural placement of payload modules on the depot ship}

In order to place the payload modules, there should be a free space on the upper deck, decks and platforms of the hull, as well as on superstructure decks and their side surfaces.

The limiting for a promising vessel is not the load equation, but the capacity equation in the form of a balance of areas $[1,4]$.

For a traditional hydrodynamic scheme with one hull, the need to increase the potential of a single vessel leads to an increase in the size, and, consequently, the displacement within a given class of vessels.

This is due to the fact that with an increase in displacement, the displacement utilization coefficient in terms of payload and the capacity utilization coefficient in terms of useful area increase.

A preliminary estimate of the displacement utilization coefficient for the payload can be obtained using the simplest 3 -term weight equation:

$$
\eta_{G \Gamma P}=\left(D-A * D-B * D^{2 / 3}\right) / D=(1-A)-B / D^{1 / 3}
$$

$\eta_{G \Gamma P}$ - coefficient of the displacement utilization by payload; $D$ - full displacement; $A, B-$ load meter coefficients (positive constants).

Formula (1) proves that with an increase in the vessel dimensions (its displacement), the utilization coefficient for the payload increases. 
The area available for the placement of the elements of the vessel subsystems includes the areas of the upper deck sections without superstructures $S_{U D}$, the areas of decks and platforms in the hull $S_{H}$, as well as the areas of the superstructure decks $S_{S}$.

The values of the indicated areas can be expressed in fractions of the area of the design load waterline. Then the sum of available areas $S_{\Sigma}$ will be equal to:

$$
S_{\Sigma}=S_{U D}+S_{H}+S_{S}=k_{\Sigma} * S_{U D H S}
$$

$k_{\Sigma}$ - numerical coefficient.

The general view of the equation of capacity in areas is the following:

$$
\sum_{j=1}^{J} \alpha_{j} L_{j} B_{j}+\sum_{j H=1}^{J_{H}} L_{H j} B_{H j}=\sum_{j=1}^{J} \sum_{i=1}^{I} n_{j i} k_{T j i} S_{T i}+\sum_{j H=1}^{J_{H}} \sum_{i=1}^{I} n_{H j i} K_{T H j i} S_{T i},
$$

$\alpha_{j}, L_{j}, B_{j}$ - block coefficient, length and width of the j-th deck;

$J$ - the number of decks on the vessel, including the upper one;

$L_{H j}, B_{H j}$ - length and width of the j-th superstructure deck;

$J_{H}$ - the number of superstructures;

$S_{T i}$ - the area required to place the i-th module, taking into account gaps, maintenance areas, communications, etc.;

$k_{T j i}, k_{T H j i}$ - the coefficient of the presence of a module of the i-th type on the $\mathrm{j}$-th deck or on the $\mathrm{j}$-th superstructure, respectively $\left(k_{T j i}=1\right.$ - the $\mathrm{i}$-th module is present on the $\mathrm{j}$-th deck, $k_{T j i}=0$ otherwise);

$n_{j i}, n_{H j i}$ - the number of modules of the i-th type, placed on the corresponding deck.

The equation can be transformed using the parabolic representation of the sailing line at the waterlines and assuming that the length and breadth of the vessel are constant at all waterlines. It is also assumed that the lengths of all superstructures are constant and can be expressed in fractions of the vessel's length, and their widths coincide:

$$
L B\left(\sum_{j=1}^{J}\left(\frac{Z_{j}}{T}\right)^{\left(\frac{\alpha}{\delta}-1\right)}+\sum_{j H=1}^{J H} l_{H j}\right)=\sum_{j=1}^{J} \sum_{i=1}^{I} n_{j i} k_{T j i} S_{T i}+\sum_{j H=1}^{J_{H}} \sum_{i=1}^{I} n_{H j i} K_{T H j i} S_{T i},
$$

$\delta, \alpha, L, B$ - block coefficients, coefficients of fullness of the design load waterline, length and breadth of the vessel;

$Z_{j}-\mathrm{z}$-coordinate of the $\mathrm{j}$-th deck;

$T$ - draught;

$l_{H j}=L_{H j} / L-$ the relative length of the $\mathrm{j}$-th superstructure.

Thus, using formulas (2-4) it is possible to make a preliminary assessment of the requirements for the placement of payload modules at the early stages of vessel design.

Let us assume that in the problem of optimizing the geometric structure of modules, there are only $N$ components, each of which is characterized by a mounting volume $v_{i}$. Components are combined into the $K$ information systems. The payload can be grouped into the $M$ modules. There are $M$ mounting zones to place the modules. Each zone has a volume $W_{j}$. Crossing the mounting zones is not allowed.

The set of component indicators are placed in the zone $\mathrm{m}$ as $I_{m}$. The distance between the mounting zones is set by the matrix $R=\left\|r_{i j}\right\|$. Due to the mounting conditions, the distances between the components cannot exceed the maximum permissible values set by the matrix $P=\left\|\rho_{i j}^{*}\right\|$. For functionally unrelated components $\rho_{i j}^{*}=\infty$. For components placed in the same mounting area $\rho_{i j}^{*}=0, i, j \in I_{m}$. For components placed in the $\mathrm{p}$-th and q-th mounting zones

$$
\rho\left(x_{i}, x_{j}\right)=r_{p q}, i \in I_{p}, j \in I_{q}
$$


Thus, the following set of constraints is formed:

$$
\begin{aligned}
\sum_{i \in I_{m}} v_{i} & \leq W_{m}, m=1, \ldots, M, \\
\rho_{i j} & \leq \rho_{i j}^{*}, i, j=1, \ldots, N .
\end{aligned}
$$

The minimized communication length of the modules $\min \sum_{i j} \rho_{i j}$ is taken as a criterion. Thus, this problem is a combinatorial problem of finding a solution on a graph, and can be solved using the branch and bound algorithm $[1,5,6]$.

\section{Estimation of cost parameters of placing payload modules}

The following methods are used:

- inverse problem method;

- comparative method;

- indexing method;

- method of expert evaluations.

The source data in this method include:

- basic model (analog vessel not equipped with payload modules);

- the cost of the basic model;

- year of creation of the basic model;

- the composition of the basic model payload;

- the cost of the payload;

- years of payload creation;

- expert assessments on the values of the applied coefficients.

Calculation algorithm:

- Selection of an analogue vessel (prototype);

- Determination of the cost and year of production of an analog vessel;

- Determination of the payload composition and its cost;

- Determination of the cost of the hull without payload (the payload is taken into account, potentially suitable for upgrading to modules):

$$
C_{c}=C_{c}^{S S C}-\sum_{i=1}^{N} C_{i}^{S S C}
$$

$C_{c}$ - the cost of a vessel built according to the classical principle, excluding the cost of modules;

$C_{c}^{W M S E c_{-}}$the cost of a vessel built according to the classical principle, taking into account the cost of the payload;

$C_{i}^{W M S E C_{-}}$the cost of the i-th payload complex (classic);

$N$ - the number of payload complexes (classic).

Calculation of the cost of a vessel's hull adapted to work with a modular payload:

$$
C_{m}=C_{c} * F_{c} * I_{p}
$$

$C_{m}$ - the cost of a vessel adapted to work with a modular payload, excluding the cost of modules;

$C_{c}-$ the cost of a ship built according to the classical principle, excluding the cost of the payload;

$F_{c}$ - complexity factor;

$I_{p}$ - producer price index. 


\subsection{Determination of the complexity factor}

The complexity factor reflects the total number of technical solutions required to create a certain class of vessels with given technical and operational properties, based on the set tasks. [7, 8]

Bringing the cost of the hull of an analogue vessel to the design year using producer price indices.

Since the calculation is based on the prices of the basic payload models already produced earlier, deflator indices are used in order to bring their cost to the current or forecast period.

$$
I_{p}=\prod_{i=1}^{N}\left(1+I_{p i}\right)
$$

$I_{p}$ - producer price index.

$I_{p i}$ - producer price index for the i-th year;

$N$ - designation of the current year.

Calculation of the cost of the payload, adapted to work in modules [9].

$$
C_{i}^{W M S E m}=C_{i}^{W M S E C} * C_{N i} * I_{p},
$$

$C_{i}^{\text {WMSEm }}$ - the cost of the $\mathrm{i}$-th payload complex, adapted to work in the module;

$C_{i}^{W M S E C}$ - the cost of the i-th payload complex (classic);

$C_{N i}$ - the coefficient of novelty of the i-th payload complex, adapted to work in the module; $I_{p}$ - producer price index;

\subsection{Determination of the novelty factor}

The novelty factor takes into account the level of superiority of the characteristics of the developed model of the payload over the selected analogue [9-11].

This factor is introduced for the cost estimate of the installed payload modules and is determined by an expert evaluation based on the classification.

Full-scale "revolutionary" development of a payload model. It is a development option that suggests changes in the structural and layout solutions of a promising model in comparison with an analogue model (basic model) as a result of which a set of scientific and technical innovations affecting the model as a whole is implemented and involves fundamentally new properties (capabilities).

"Evolutionary" option for the development of a payload model. It is a development option that suggests changes in the structural and layout solutions of a promising model in comparison with an analogue model (basic model) as a result of which a set of scientific and technical innovations affecting the model as a whole is implemented but without fundamentally new properties (capabilities).

"Deep" modernization of the payload model. It is the replacement of a significant part (more than 50\%) of the main subsystems of the model with subsystems of a new generation, in which a set of scientific and technical innovations is implemented, leading to a significant improvement in technical characteristics.

"Average" modernization of the payload model. It is the replacement of individual subsystems (from 15 to $50 \%$ ) of the model with subsystems of a new generation, in which a set of scientific and technical innovations is implemented, leading to an improvement in tactical and technical characteristics. It occupies an intermediate position between "deep" and "minor" modernization.

"Minor" modernization of the payload model. It is the modification of its individual subsystems (less than 15\%), leading to a slight improvement in technical characteristics. 
Calculation of the total cost of the vessel with payload modules:

$$
C_{m}^{W M S E m}=C_{m}+\sum_{i=1}^{N} C_{i}^{W M S E m},
$$

$C_{m}^{W M S E m}$ - the cost of a vessel with payload modules;

$C_{m}$ - the cost of the vessel's hull, adapted to work with modules, excluding the payload;

$C_{i}^{\text {WMSEm }}$ - the cost of the i-th payload complex, adapted to work in the module;

$\mathrm{N}$-the number of payload complexes adapted to work in the module.

\section{Conclusion}

The article discusses methods for preliminary estimation of the placement of replaceable payload modules on scientific vessels during research design. The method for developing the constructive placement of payload modules on a depot ship and the assessment of the cost parameters of the placement are proposed. The in-depth evaluations obtained when using the developed methods can be used to formulate technical requirements for promising scientific vessels.

\section{References}

1. A.I. Borovkov, L.B. Maslov, K.S. Ivanov, et al., Int. J. of Mech. and Prod. Eng. Res. and Dev. (IJMPERD) 10, 14861 (2020)

2. D. Vuksanović, J. Ugarak, D. Korčok, Sinteza, Int. Sc. Conf. on ICT and E-Bus. Rel. Res. 293 (2016)

3. Z. Chen, S. Cao, Z. Mao, Energies 11, 28 (2018)

4. A.D. Karpov, A.A. Zhilenkov, D. Lisitsa, IEEE Conference of Russian Young Researchers in Electrical and Electronic Engineering (EIConRus) 887 (2017) doi: 10.1109/EIConRus.2017.7910697

5. Z. Chen, S. Cao, Z. Mao, Energies 11, 28 (2018)

6. A.A. Zhilenkov, D. Denk, IEEE Conference of Russian Young Researchers in Electrical and Electronic Engineering (EIConRus) $1104 \quad$ (2017) doi: 10.1109/EIConRus.2017.7910748

7. A. Al-Dulaimi, S. Zabihi, A. Asif, A. Mohammadi, Comput. Ind. 108, 186-96 (2019)

8. A.V. Ivanov, A.A. Zhilenkov, IEEE Conference of Russian Young Researchers in Electrical and Electronic Engineering (EIConRus) $\mathbf{8 9 0}$ (2018) doi: 10.1109/EIConRus.2018.8317231

9. A. Bhardwaj, W. Di, J. Wei, Deep Learning Essentials: Your hands-on guide to the fundamentals of deep learning and neural network modeling (Packt Publishing Limited, Birmingham, 2018)

10. J.T. Barron, Proc. of the IEEE Conf. on Computer Vision and Pattern Recognition (CVPR) (2019)

11. D. Lisitsa, A.A. Zhilenkov, IEEE Conference of Russian Young Researchers in Electrical and Electronic Engineering (EIConRus) 926 (2017) doi: 10.1109/EIConRus.2017.7910708 